\title{
Oscillator-Type Active-Integrated Antenna: The Leaky-Mode Approach
}

\author{
Guang-Jong Chou and Ching-Kuang C. Tzuang, Senior Member, IEEE
}

\begin{abstract}
This paper describes basic research carried out to design a microwave source module employing the concept of an active-integrated leaky-mode antenna. The novel activeantenna source module utilizes a microstrip as the radiating element while adopting uniplanar technology for the active circuit design. The microstrip is operated in the first higher order odd mode, which is a leaky mode, and excited by a proximitycoupled center-fed slotline on the same surface of the uniplanar microwave-integrated circuit. The measured performance of an $X$-band transmission-type injection-locked active-integrated antenna source module demonstrated that such a design approach was suitable for linear array integration for quasi-optical power combining. The harmonic-balance (HB) analysis of the proposed active-integrated antenna agrees with the measurements in both free-running frequency and power level. The measured radiation patterns of the active-integrated antenna also agree well with the theoretical predictions.
\end{abstract}

\section{INTRODUCTION}

A CTIVE-INTEGRATED antennas have come into prominence in recent years since mature monolithic microwave-integrated circuit technology is commensurate with antenna integration in a variety of transceiver applications, particularly at lower millimeter wave frequencies [1]-[3]. An active-integrated antenna, by definition, integrates the active microwave circuits and the radiating element on the same substrate, thereby reducing transition and transmission losses [1]. A complete monolithic integration using the active-integrated antenna approach can make the system very compact, low cost, and reliable. Active-integrated antennas, according to their functions, can be classified into three categories: oscillators, amplifiers, and frequency converters [1]. There is great interest in using an oscillator-type activeintegrated antenna for generating microwave and millimeter wave signals. Applications reside in many areas such as spatial power combining [4]-[6], radar [7], and intelligent vehicle guidance [3].

The oscillator-type of active-integrated antenna is comprised of a radiating element, feed, and oscillator. To date, the microstrip patch antenna has been the most commonly used radiating element due to its lightweight, planar structure and ease of handling. It suffers, however, from the presence of surface wave modes and an increasing cross-polarization level at the millimeter waves [8], [9]. There are other types of planar antennas implemented in various active-integrated

Manuscript received March 19, 1996; revised August 26, 1996. This work was supported by the National Science Council, R.O.C., under Grants NSC 85-2213-E009-003 and C85099.

The authors are with the Institute of Electrical Communication Engineering, National Chiao Tung University, Hsinchu 300, Taiwan, R.O.C.

Publisher Item Identifier S 0018-9480(96)08509-2. antennas, e.g., the slot antenna [10], notch antenna [5], and leaky-wave antennas [2], [11]. The leaky-wave antenna is a class of traveling-wave antenna which can be implemented by periodic structure or invoking the leaky mode of a guided wave device. The latter possesses the characteristics of having a narrow beam, frequency scanning, and ease of matching at the excitation port. As a result, numerous studies have been carried out involving linear antenna-array integration using leakywave antenna elements [12]. The radiation characteristics of such a leaky-wave antenna can be fully understood by obtaining its complex propagation constant which becomes the fundamental parameter for the antenna design. The leakywave antenna described in this paper is a microstrip line which is planar and leaks in the form of the first higher order odd mode [13].

This paper details the analysis and design of an activeintegrated leaky-mode antenna source module in which the preliminary version showed good results for use in quasioptical power-combiner design [11]. The idea of exciting the microstrip's first higher order leaky mode on one side of the substrate while retaining all of the merits of the uniplanar microwave-integrated circuits [14] on the other side becomes quite natural when a slotline underneath the middle of the microstrip is employed as an efficient antenna feed. The active microwave network is thereby separated from the radiating element except for the coupling circuit [15]. Active CPW circuits have several attractive features:

1) reduced cross coupling;

2) a ground connection that does not require via holes;

3 ) ease of device mounting in series and shunt configurations;

4) low radiation loss.

\section{ANALYSIS, DESIGN, AND CHARACTERISTICS \\ OF A MICROSTRIP LEAKY-MODE ANTENNA}

In contrast to the well-known dominant mode propagating in a microstrip, the first higher mode interests us here. It leaks in the forms of space wave or surface wave. The latter is the lowest order $\mathrm{TM}_{0}$ surface wave with a zero cutoff which is often weakly excited. Therefore, the space wave is dominant in the case of the microstrip leaky-mode antenna [16]. This is desirable since most electromagnetic energy will propagate in the intended direction rather than dissipating in the substrate. By obtaining the dispersion characteristics of the microstrip's leaky mode, the radiation pattern of the antenna can be readily determined. 


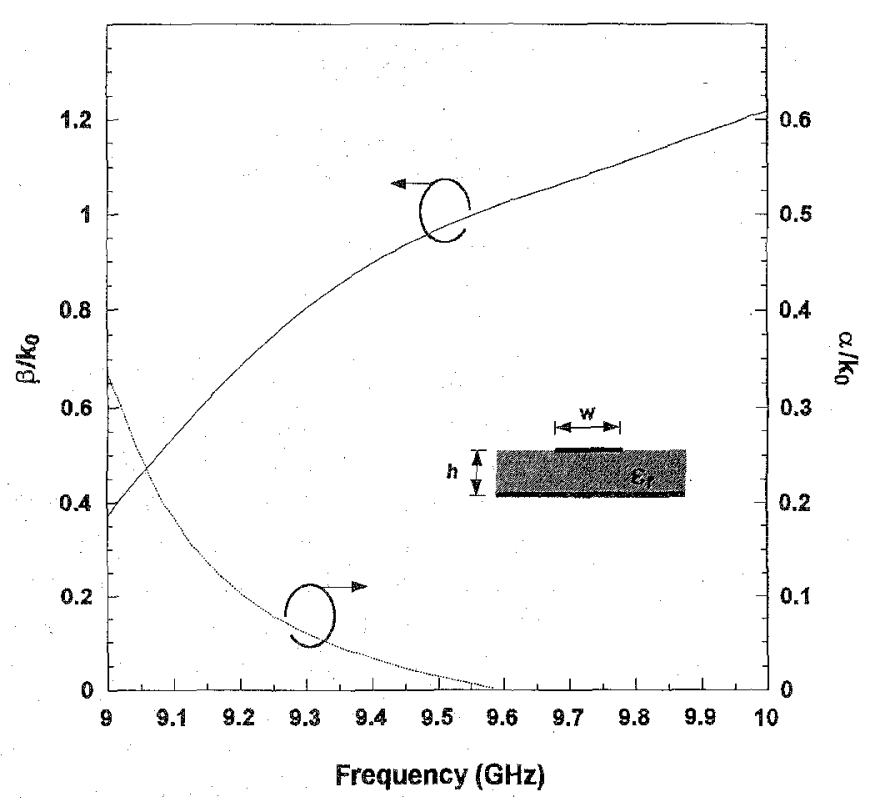

Fig. 1. Normalized complex propagation constant of the first higher mode for the particular microstrip leaky-mode antenna. $h=.635 \mathrm{~mm}, w=4.572$ $\mathrm{mm}$, and $\varepsilon_{r}=10.2 . k_{0}$ is the free-space wave number.

\section{A. Dispersion Characteristics of the Leaky-Mode Antenna}

The radiation characteristics of a leaky-wave antenna can be derived by obtaining the corresponding complex propagation constant $k_{z}$, where $k_{z}=\beta-j \alpha . \beta$ is the phase constant and $\alpha$ the attenuation constant. Positive $\alpha$ means that the guided power gets attenuated as the leaky mode radiates with a main beam position at $\theta_{0} \cong \cos ^{-1}\left(\beta / k_{0}\right)$, measured from the longitudinal $z$-axis. Fig. 1 plots the normalized dispersion curves of the first higher mode in the leakage region for a particular microstrip on a 25-mil-thick RT/Duroid 6010 substrate. The substrate's permittivity is chosen close to that of GaAs in the hope that the performance demonstrated in the paper can be obtained in a GaAs monolithic-microwave integrated circuit (MMIC) without much modification. The complex propagation constants are obtained rigorously by the spectraldomain approach [17]. The particular design leaks in a fan beam fashion below a threshold frequency at approximately $9.58 \mathrm{GHz}$. When one plots the beam position angle $\theta_{0}$ and leakage rate derived from $\alpha$ against the frequency as shown in Fig. 2, the radiation characteristics of the active-integrated leaky-mode antenna source module are largely known.

\section{B. Radiation Pattern}

The field equivalence principle is applied to derive the far field radiation pattern of the microstrip leaky-mode antenna. Fig. 3 shows the model of the structure. There are two equivalent magnetic surface currents along the microstrip's two edges, where two apertures, each of length $L$ and height $h$, are separated by a distance $w$ equal to the microstrip's width. The electric fields $\vec{E}_{1}$ and $\vec{E}_{2}$ at the apertures can be assumed to be exponentially decaying functions with an attenuation constant $\alpha$ and can be expressed as

$$
\begin{aligned}
& \vec{E}_{1}=\hat{x} E_{0} e^{-j(\beta-j \alpha) z} \\
& \vec{E}_{2}=-\hat{x} E_{0} e^{-j(\beta-j \alpha) z}
\end{aligned}
$$



Fig. 2. Beam position angle $\theta_{0}$ and leakage rate of the particular microstrip leaky-mode antenna in Fig. 1.

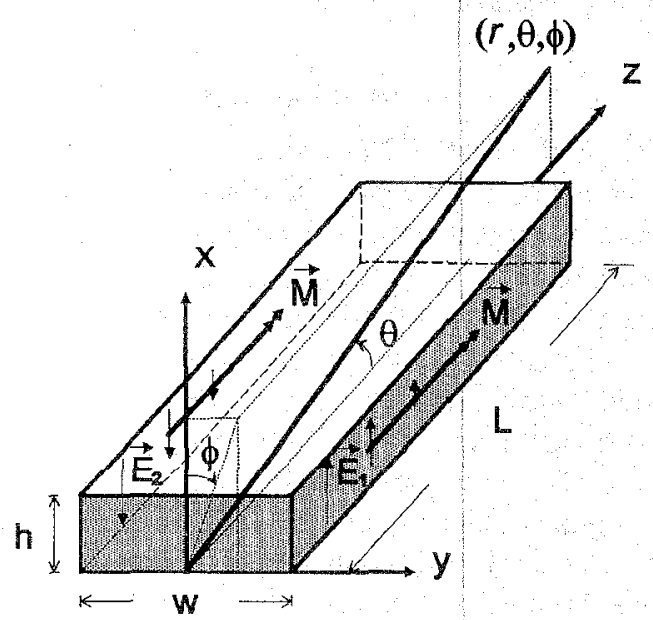

Fig. 3. Geometry and coordinate system for deriving the far-zone radiation pattems of a microstrip leaky-mode antenna using Huygen's principle. The microstrip leaky-mode antenna is of width $w$, height $h$, and length $L$.

where $E_{0}$ is an arbitrary constant. By applying Huygen's principle to the aperture plane, we obtain the equivalent magnetic surface currents. Thus, the far-zone electric fields of the leaky-mode antenna with length $L$ [18] can be written as

$$
\begin{aligned}
E_{r} \cong & E_{\theta} \cong 0 \\
E_{\phi} \cong & -j E_{0} \frac{k h e^{-j k r}}{\pi r}\left\{\sin \theta\left[\frac{\sin (X)}{X}\right]\left[\frac{e^{Z L}-1}{Z}\right]\right\} \\
& \cdot \cos \left(\frac{k w \sin \theta \sin \phi}{2}\right)
\end{aligned}
$$

where

$$
\begin{aligned}
& X=k h \sin \theta \cos \phi \\
& Z=j(k \cos \theta-\beta)-\alpha
\end{aligned}
$$

and $k=2 \pi / \lambda$ are the wave numbers. Equation (2) assumes that there is no reflection from the far end of the leaky-mode 


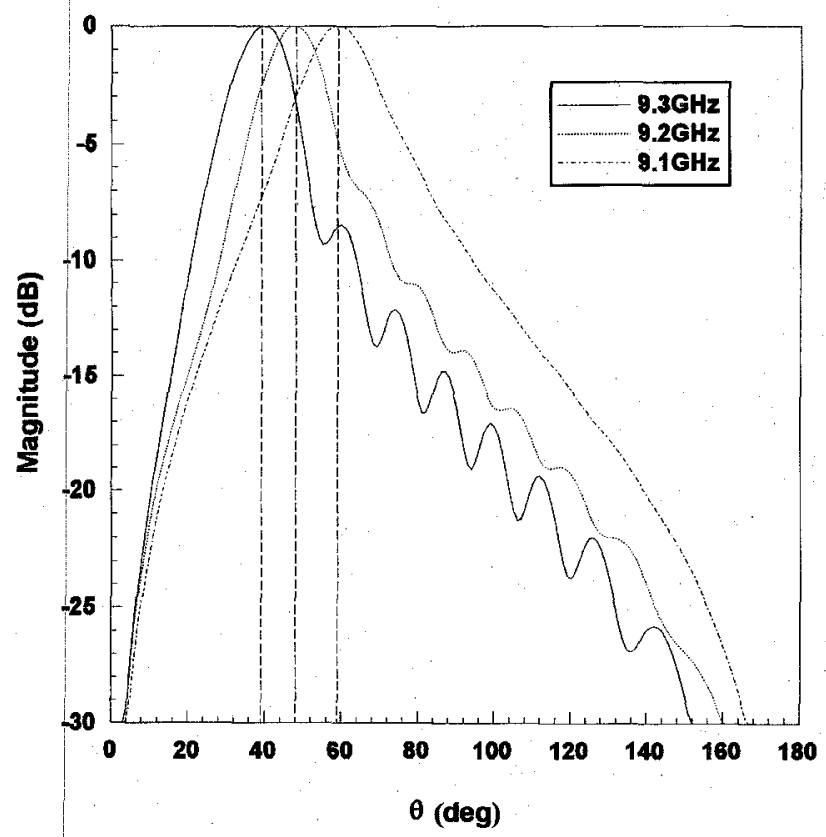

Fig. 4. Theoretical $H$-plane ( $x-z$ plane) radiation patterns of the microstrip leaky-mode antenna against the elevation angle for three different frequencies. The antenna length is $150 \mathrm{~mm}$ long.

antenna and clearly shows that a fan beam polarized in the $\phi$ direction with its maximum located in the $\phi=0^{\circ}$-plane, which is the H-plane of the microstrip leaky-mode antenna. Along this H-plane, the normalized radiation amplitude patterns of the finite-length microstrip leaky-mode antenna derived from (2) are plotted in Fig. 4 for operating frequencies at 9.1, 9.2, and $9.3 \mathrm{GHz}$, respectively. Also shown are the frequency scan characteristics of the antenna at $9.3,9.2$, and $9.1 \mathrm{GHz}$ where the beam position varies from $39^{\circ}$ through $48^{\circ}$, to $59^{\circ}$ as the $3-\mathrm{dB}$ beamwidth is widened from $17.5^{\circ}$ through $18^{\circ}$, and to $22.5^{\circ}$, respectively. Comparing these numbers for the beam position angles with the corresponding data of Fig. 2 , the difference is less than $2^{\circ}$, caused by the finite length $(L)$ of the antenna. When $\alpha$ is small, and $L$ is large enough, the beam position angles derived from (2) and those predicted in Fig. 2 are almost the same.

\section{The Proximity-Coupled Feed and RPA Measurement}

Fig. 5 shows the close-up view of the proximity-coupled slotline feed of the microstrip leaky-mode antenna which is flipped over to the bottom side of the figure. The slotline feed may extend to the end of the leaky-wave antenna [19]. In Fig. 5, short-circuited slotline feed is used on top of the microstrip. We may improve the coupling and prevent the dominant mode from being excited by employing the slotline of a length greater than a half wavelength. In addition to the slotline feed, a typical CPW cross for the CPWto-slotline transition is applied to the feeding structure for ease of interface with the vector network analyzer (VNA) as well as the active uniplanar MMIC with CPW ports. Each transmission line extends a quarter wavelength beyond the crossing point where the slotline is terminated short, and the CPW is terminated open. The design of such a CPW cross has been well developed and investigated [20].

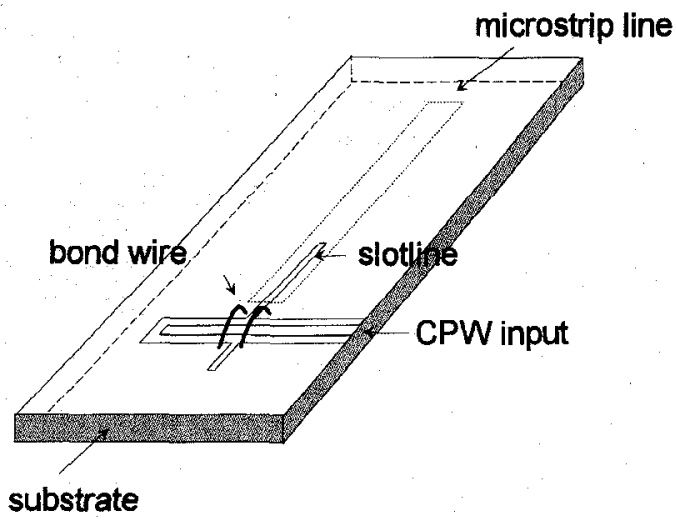

Fig. 5. The close-up view of the proximity-coupled leaky-mode antenna employing the center-fed slotline.

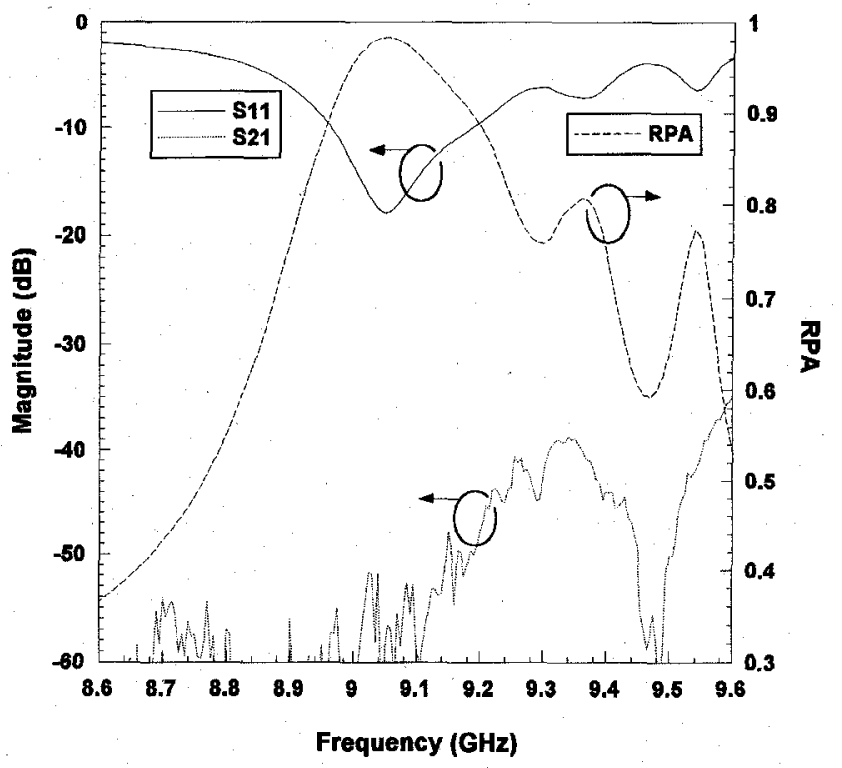

Fig. 6. Measured $S$ parameters and RPA of the CPW-fed microstrip leaky-mode antenna with two feeds. The antenna length $L=150 \mathrm{~mm}$.

The solid line of Fig. 6 shows that good input matching to the $50-\Omega$ reference impedance is obtained with input reflection coefficients kept below $-10 \mathrm{~dB}$ for the frequency range of 9.0-9.2 GHz. The surviving leaky-mode energy at the end of traveling along the microstrip line will be converted to the CPW mode at the receiving end of the antenna with the same CPW cross at the input port. The measured transmission parameter $S_{21}$ indicates that a negligible amount of leaky-mode energy reaches the receiving end, although the magnitude of $S_{21}$ is relatively high between 9.1 and $9.6 \mathrm{GHz}$ (just below the cutoff frequency). We may compute the value for the relative power absorbed (RPA $=1-\left|S_{11}\right|^{2}-\left|S_{21}\right|^{2}$ ) from the backto-back connected microstrip leaky-mode antenna and plot the results in Fig. 6 [21]. The RPA accounts for the energy loss in the microstrip leaky-mode antenna and two CPW crosses. It also accounts for ohmic losses due to finite metal conductivity, substrate dissipation, undesired surface wave excitation, and radiation from the slotline, etc.

We have conducted a near-field measurement of the leakywave antenna. The radiation contour pattern illustrates that the 


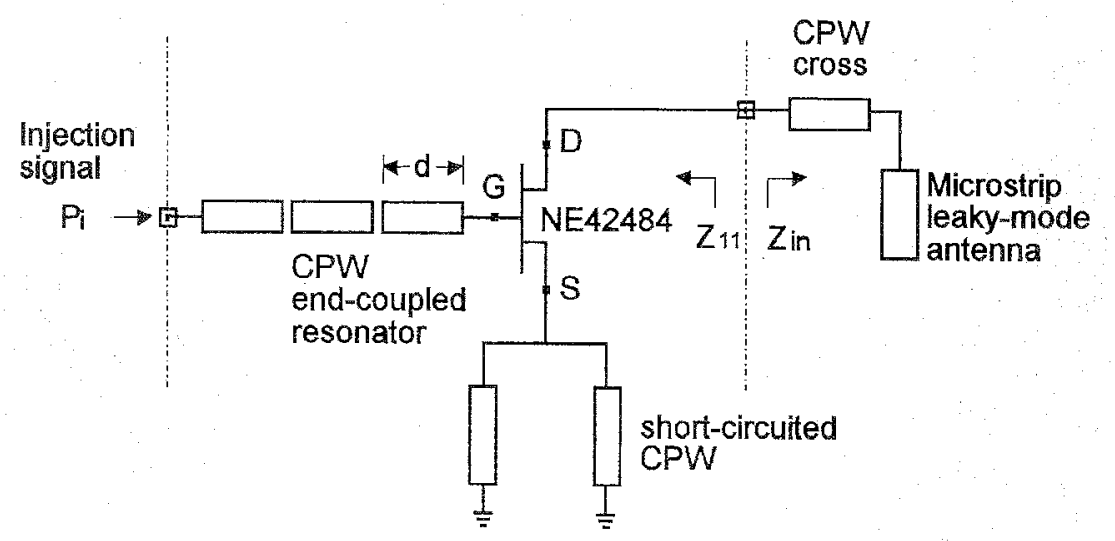

Fig. 7. Simplified schematic diagram for the quasi-optical oscillator.

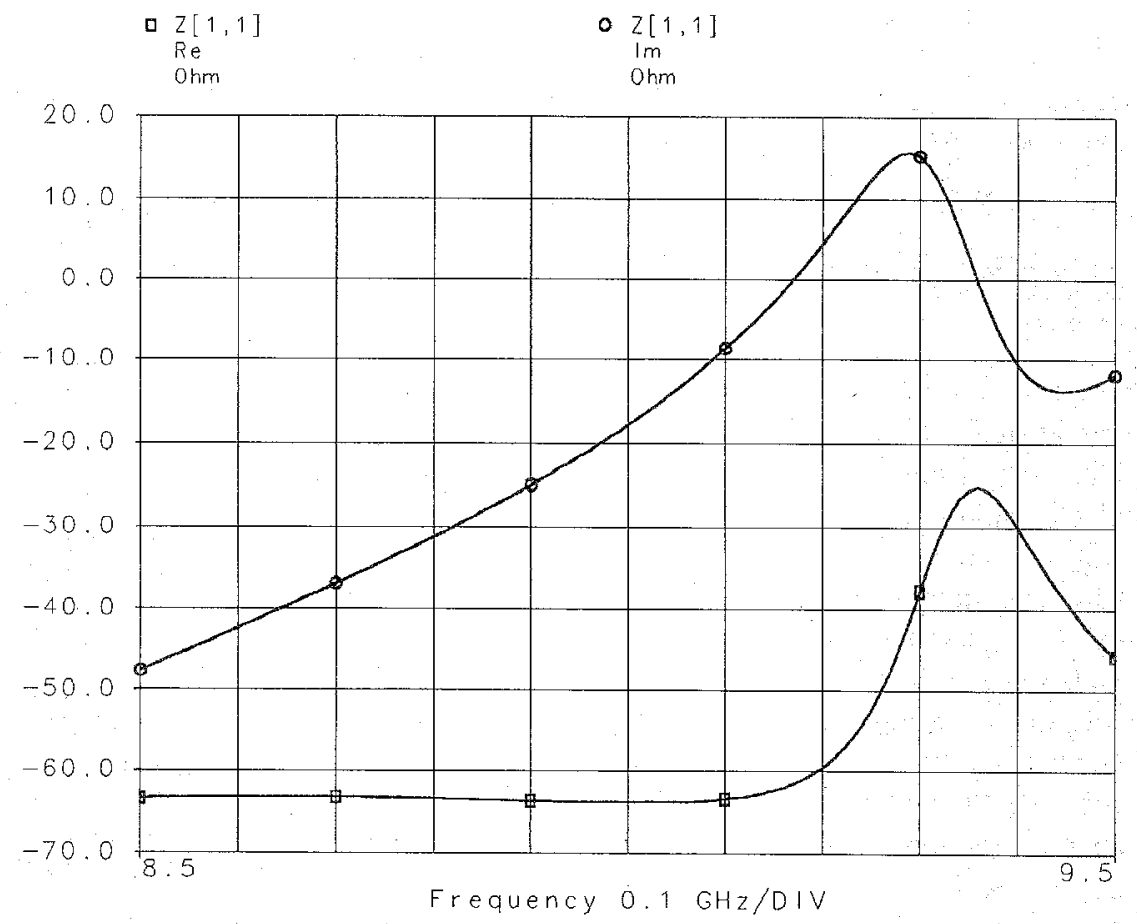

Fig. 8. Small-signal simulation of input impedance at the drain port of the oscillator.

main energy leakage is indeed the leaky wave emanating from the microstrip line at the predicted beam position, whereas the only other noticeable leakage is from the slotline radiation showing $-14 \mathrm{~dB}$ down with respect to the main beam. The surface wave leakage is not detected in the near-field measurement. Thus, we may conjecture that the energy loss associated with the RPA is primarily leaky-wave radiation if the conductor and dielectric losses can be neglected. Fig. 6 shows that the RPA can be as high as 0.98 , implying that almost all of the energy is radiated with good antenna-input matching.

\section{DESIGN AND ANALYSIS OF A QUASI-OPTICAL OSCILLATOR}

The CPW input port of the microstrip leaky-mode antenna of Fig. 5 facilitates the interface to the uniplanar MMIC. Such an arrangement directly leads to the design of the quasi-optical oscillator to be described here. Fig. 7 shows the simplified schematic diagram of the injection-locked quasi- optical oscillator. An NE42484 low-noise HEMT is chosen as the active device. The oscillator employs a series feedback at the HEMT's source terminal to create substantial negative resistance. The injection signal is coupled into the gate terminal via CPW end-coupled resonators. Therefore the dc isolation is self-contained. The resonator's length, d (see Fig. 7), helps set the oscillation frequency. Both linear and nonlinear simulations are invoked to design the oscillator.

\section{A. Oscillator Design: Linear Analysis}

The oscillator design adopts the simplified design approach proposed by Johnson [22]. He assumed that under large-signal operation the small-signal $S$ parameters remain the same except for a reduction in the magnitude of $S_{21}$. When designing an oscillator, one's goal is to obtain the maximum output power. Hence, the modified (reduced) value of $S_{21}$ is derived by maximizing the oscillator output power. Then the modified scattering parameters are applied 


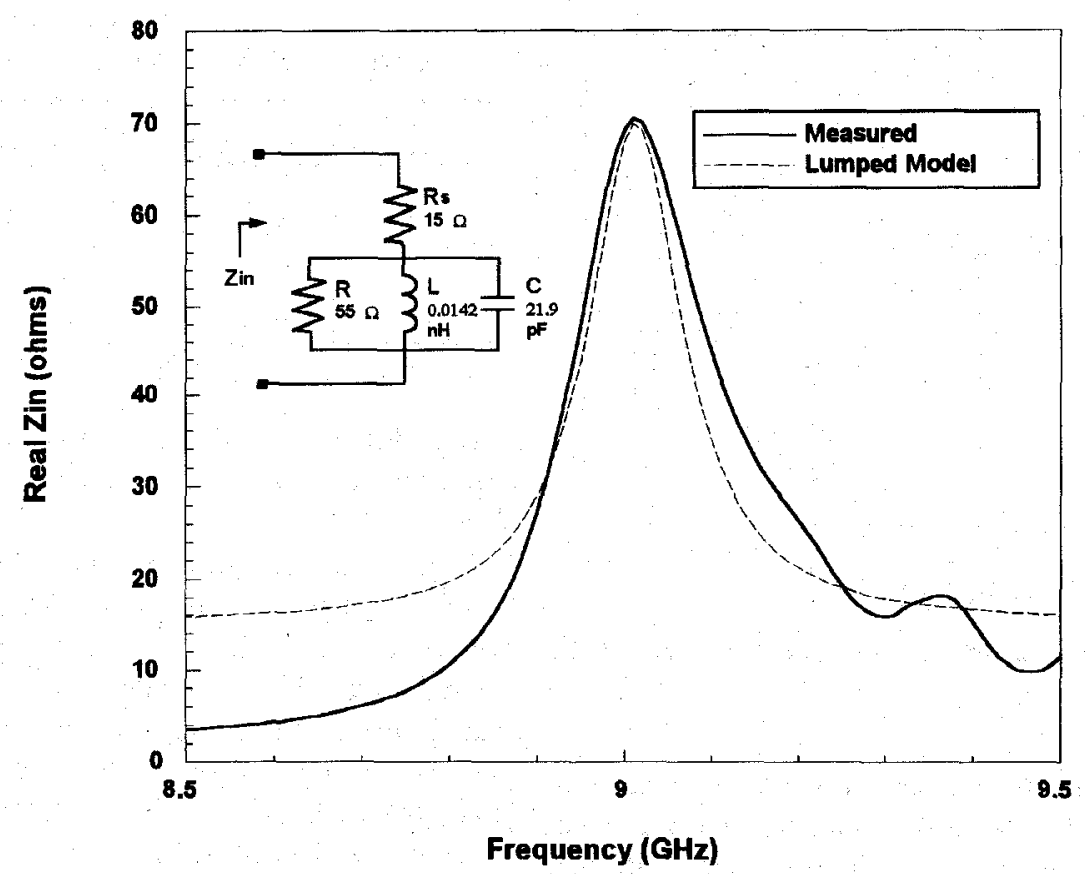

(a)

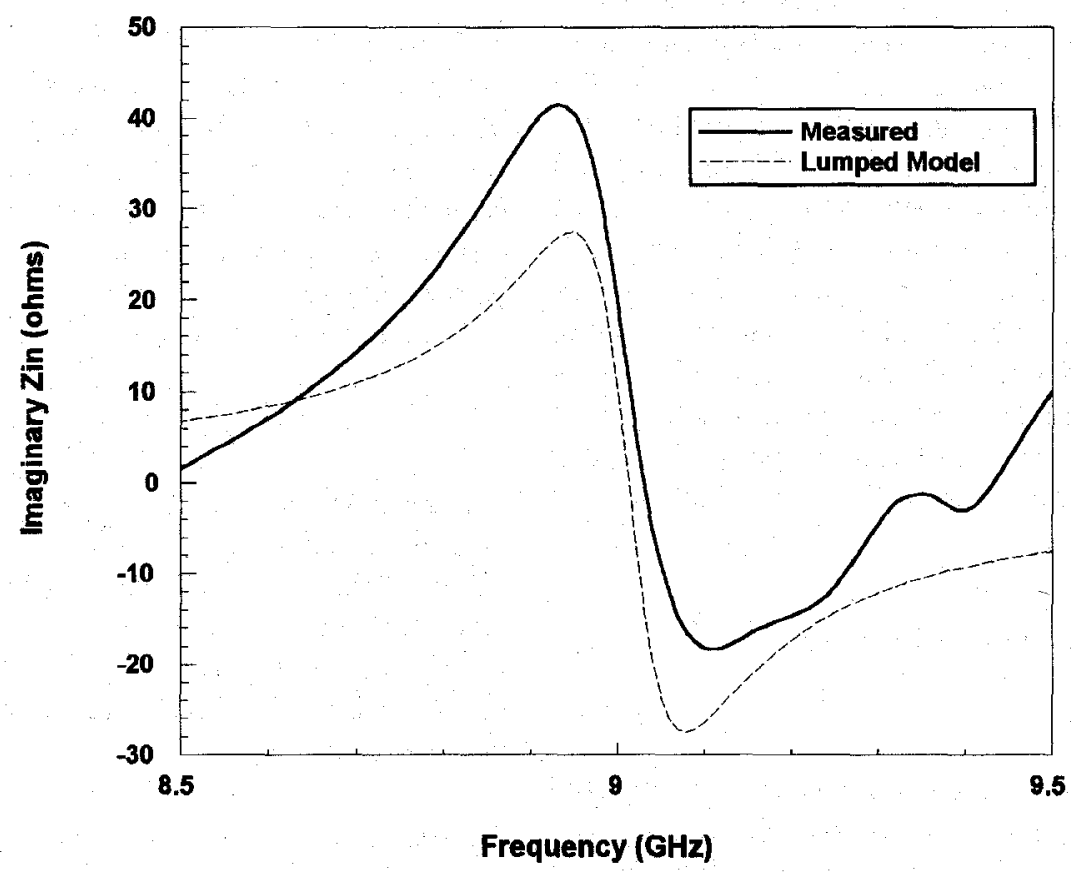

(b)

Fig. 9. Comparison of the measured and modeled input impedance of the microstrip leaky-mode antenna. (a) Real part of the input impedance and (b) imaginary part of the input impedance.

to the oscillator design using linear circuit analysis. Fig. 8 shows the oscillator output impedance $Z_{11}$ looking into the drain port with the injection port terminated at $50 \Omega$. Between 8.5 and $9.3 \mathrm{GHz}$, the reactance has a positive slope implying stable oscillation while showing negative resistance for all of the interested frequencies.

\section{B. Harmonic-Balance Analysis}

Next, the harmonic-balance method is used to predict the oscillation frequency and optimize output power. Before in- voking a Libra harmonic-balance analysis of the nonlinear oscillator, the microstrip leaky-mode antenna must be properly modeled by an equivalent one-port lumped circuit looking into the CPW-fed port of the antenna. The solid line in Fig. 9 shows the measured complex input impedance $Z_{\text {in }}$ of the CPW-fed antenna, which can be modeled as a cascade of a parallel RLC resonant circuit, and series resistor $R_{s}$ as shown in the inset of Fig. 9(a). The measured resonant frequency and slope parameter of $Z_{\text {in }}$ determine the values of $L$ and $C$ [23]. The 


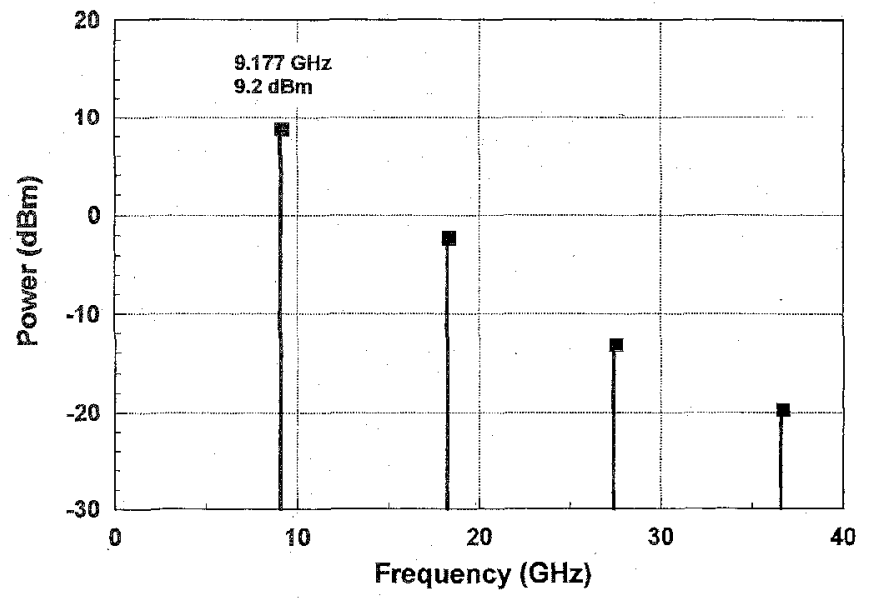

Fig. 10. Harmonic-balance analysis of the output power spectrum of the oscillator.

value of $R+R_{s}$ equals the maximum value of measured resistance. By adjusting the values of $R$ and $R_{s}$, the simulated input impedance of the equivalent circuit agrees well with the measured data.

Using the above-mentioned one-port model for the leakymode antenna, the harmonic-balance analysis sets in and shows multiple harmonics at the antenna's CPW input port. The results are plotted in Fig. 10 and indicate that the first harmonic is at $9.177 \mathrm{GHz}$ with $9.2 \mathrm{dBm}$ output power. The quasi-optical oscillator was optimized for the operation of the leaky-mode antenna over a comfortable scan range. The complete quasioptical oscillator was built on both sides of a 25 -mil-thick RT/Duroid 6010 substrate with a relative dielectric constant of 10.2. Fig. 11 shows the layout of the active-integrated antenna. In the case of a free-running situation, the near-field pickup measurement by a Tek 494AP spectrum analyzer shows that the unlocked source oscillates at $9.166 \mathrm{GHz}$, which is very close to the simulated result.

\section{MEASURED RESULTS}

\section{A. Locking Range}

It is well known that an oscillator can be synchronized by an external injection signal when the injection level is sufficient and its frequency is close enough to the free-running oscillator. Tajima and Mishima introduced the transmissiontype injection locking that separates the locking-signal input port from the power output port [24]. Thus, it does not require a circulator to isolate the input port from the output port. The injection-locking frequency range $\Delta \omega_{L}$ can be expressed as

$$
\Delta \omega_{L}=\frac{\omega_{0}}{Q_{\text {ext }}} \sqrt{\frac{P_{i}}{P_{0}}}
$$

where

$Q$ ext external $Q$-factor;

$\omega_{0} \quad$ free-running oscillator radian frequency;

$P_{0} \quad$ oscillator output power;

$P_{i} \quad$ injection power.
TABLE I

Texe Measúred Locking Range Versus injection Power Level

\begin{tabular}{c|c}
\hline Locking Range $(M H z)$ & Injection Power Level (aBm) \\
\hline 15 & -10 \\
22 & -5 \\
43 & 0 \\
\hline
\end{tabular}

Equation (3) is also known as the Adler equation. The expression of $Q_{\text {ext }}$, related to the circuit parameters, is already given in [24].

During the measurement, the injection signal from an HP $8340 \mathrm{~B}$ synthesized sweeper is connected to the CPW input port of Fig. 11, and the radiated energy is picked up by a standard gain horn connected to a spectrum analyzer via a flexible cable. Table I lists the measured locking range versus various injected power levels. For a $-10 \mathrm{dBm}$ input power level, the locking range is $15 \mathrm{MHz}$ or $1.63 \%$ of the center frequency and becomes $2.4 \%$ and $4.69 \%$ at $-5 \mathrm{dBm}$ and 0 $\mathrm{dBm}$, respectively.

\section{B. Phase Noise and Radiated Power Measurements}

Fig. 12 shows the spectrum of an injection-locked quasioptical oscillator. The injection power level is $-10 \mathrm{dBm}$ at $9.17 \mathrm{GHz}$. Phase noise of $-100 \mathrm{dBc} / \mathrm{Hz}$ at a $100 \mathrm{KHz}$ offset from the carrier is obtained by a direct phase-noise measurement out of the spectrum analyzer. Next the transmitted power of the quasi-optical oscillator is calculated by applying the Friis transmission equation [18]

$$
\frac{P_{r}}{P_{t}}=\left(\frac{\lambda}{4 \pi R}\right)^{2} G_{0 t} G_{0 r}
$$

For the sake of clarity the parameters of the measurement system and obtained results are listed in Table II. The output power of the source module is calculated as $8.6 \mathrm{dBm}$ at 9.17 $\mathrm{GHz}$. The dc bias conditions of the oscillator are $V_{d}=2$ $\mathrm{V}, V_{g s}=-0.2 \mathrm{~V}$, and $I_{d s}=32 \mathrm{~mA}$. Thus the dc-to-RF efficiency is $11 \%$. Finally, the external $Q$-factor is found to be 71.8 using (3).

\section{Radiation Pattern of the Complete Quasi-Optical Oscillator}

Fig. 13 shows the measured result for the H-plane radiation pattern and associated cross polarization at $9.17 \mathrm{GHz}$. The peak angle of the radiation pattern is $50^{\circ}$ with a beamwidth of $24^{\circ}$, and this is in very good agreement with theoretical calculations. The measured cross-polarization profile is kept approximately $-15 \mathrm{~dB}$ below the peak-radiated power throughout the measurement.

\section{Y. CONCLUSION}

This paper presents a new design approach for an activeintegrated antenna source module integrating a microstrip leaky-mode antenna and uniplanar microwave-integrated circuit on the opposite side of the same substrate. In almost every aspect, excellent agreement was obtained between the 


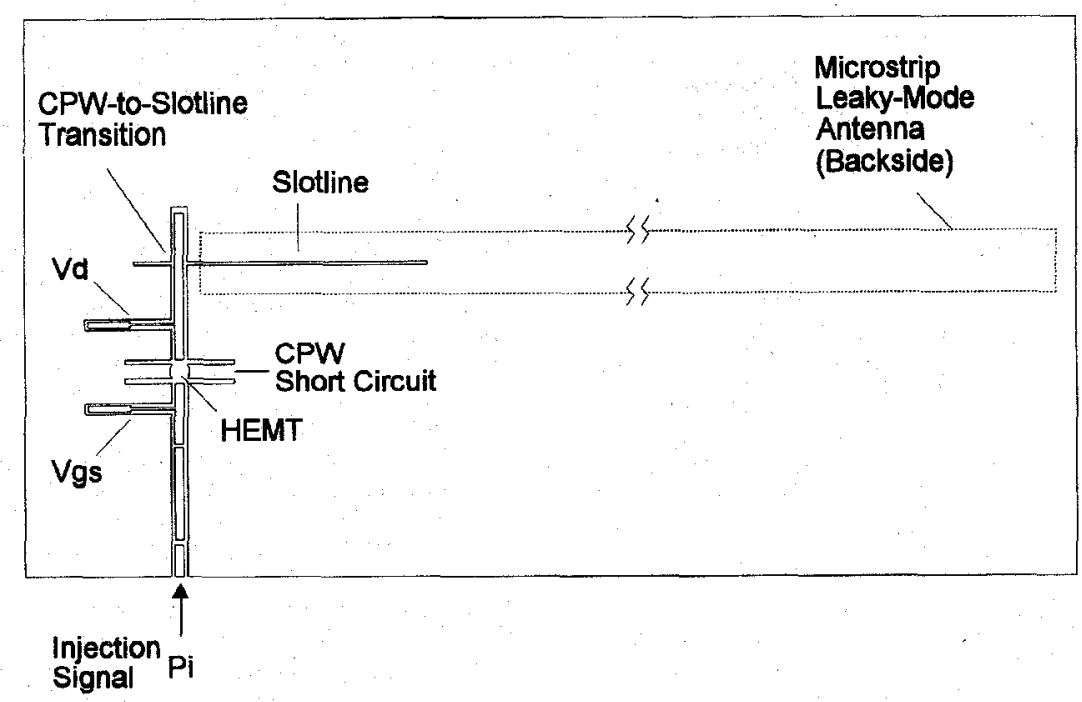

Fig. 11. Layout of the quasi-optical oscillator employing a microstrip leaky-mode antenna.

TABLE II

The Parameters of the Measurement System and Results

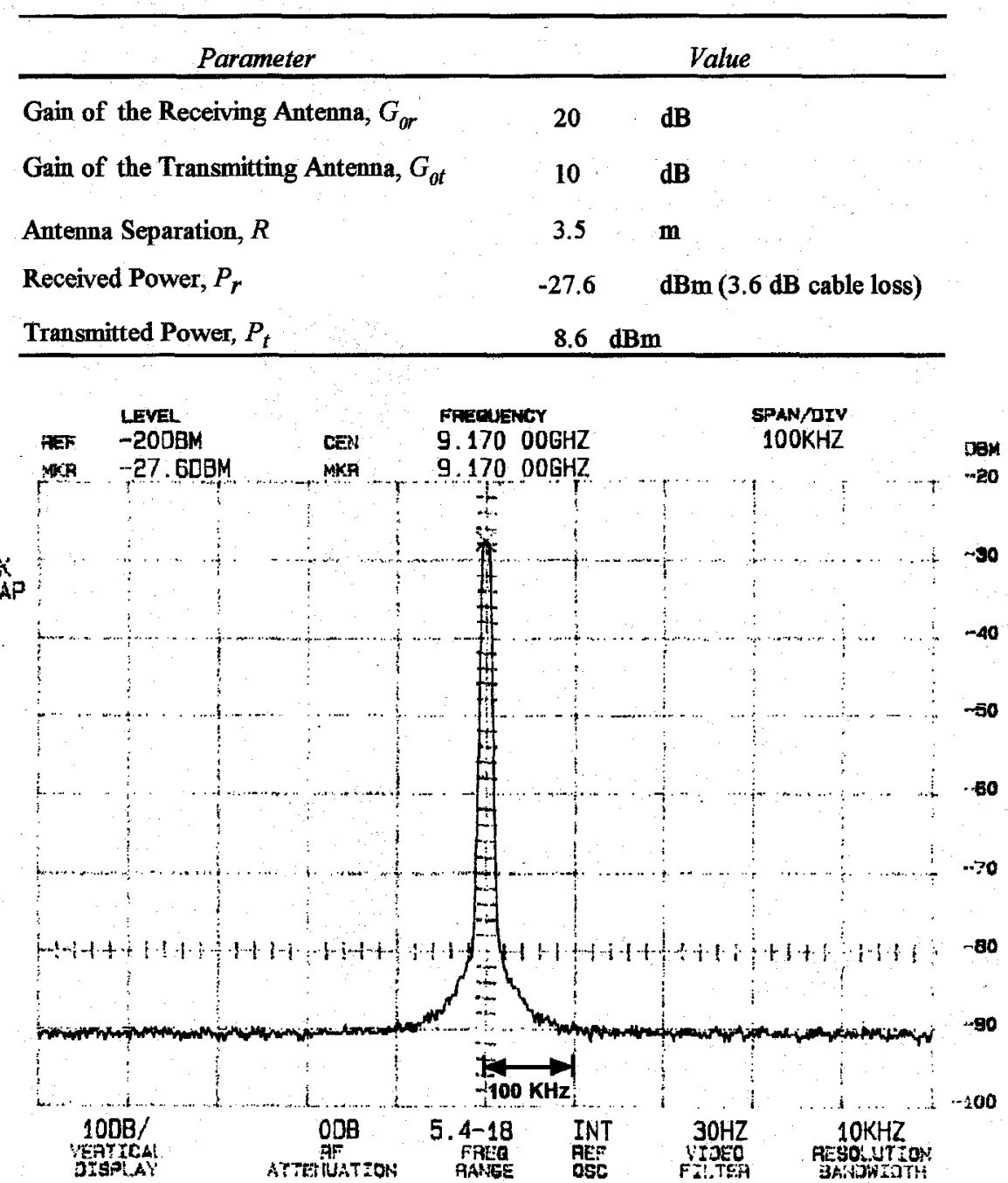

Fig. 12. Near-field power spectrum of the quasi-optical oscillator.

theoretical design of the constituent elements of the source module and corresponding measured results. The source module showed a $4.69 \%$ locking bandwidth at $0 \mathrm{dBm}$ input power, phase noise of $-100 \mathrm{dBc} / \mathrm{Hz}$ at a $100 \mathrm{KHz}$ offset from the carrier, dc-to-RF efficiency of $11 \%$, output power of $8.6 \mathrm{dBm}$ at $9.17 \mathrm{GHz}$, and cross polarization of less than $-15 \mathrm{~dB}$. 


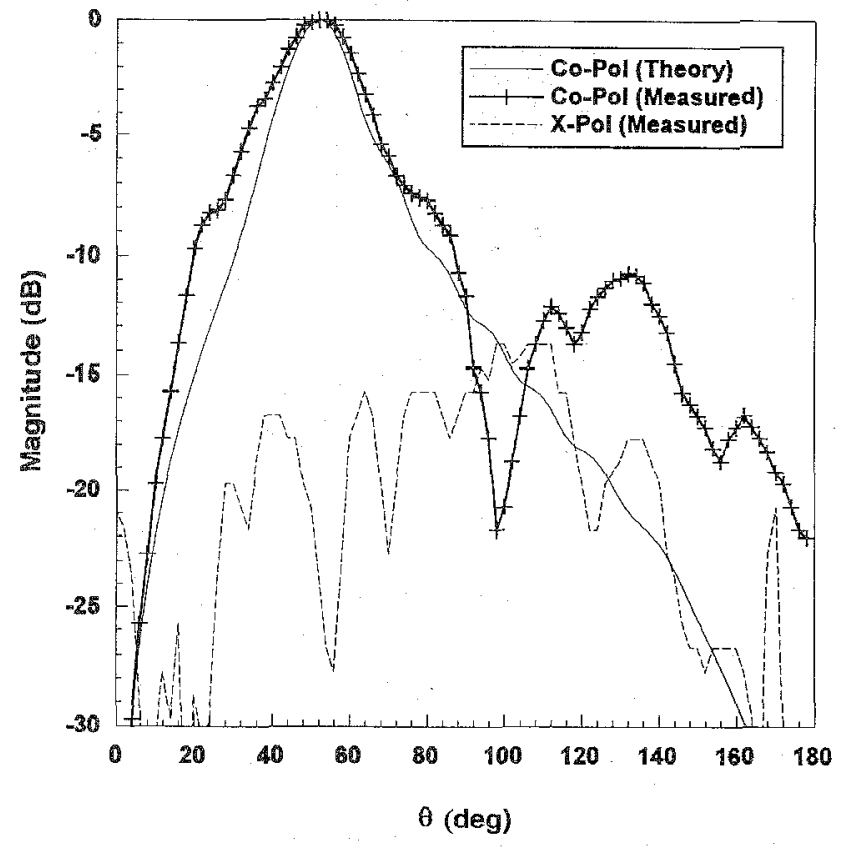

Fig. 13. H-plane patterns and cross-polarization measurements of the active-integrated microstrip leaky-mode anteana source module at $9.17 \mathrm{GHz}$.

The measured results indicate that the strategy combining a microstrip leaky-mode antenna and an active uniplanar MMIC for an active-integrated antenna design is a viable and powerful candidate for active-integrated antenna designs.

\section{REFERENCES}

[1] I. Lin and T. Itoh, "Active-integrated antennas," IEEE Trans. Microwave Theory Tech., vol. 42, pp. 2186-2194, Dec. 1994.

[2] J. Birkeland and T. Itoh, "FET-based planar circuits for quasi-optical sources and transceivers," IEEE Trans. Microwave Theory Tech., vol. 37, pp. 475-481, Mar. 1989.

[3] A. Stiller, E. M. Biebl, J.-F. Luy, K. M. Strohm, and J. Buechler, "A monolithic-integrated millimeter wave transmitter for automotive applications," IEEE Trans. Microwave Theory Tech., vol. 43, pp. 1654-1658, July 1995.

[4] R. A. York and R. C. Compton, "Quasi-optical power combining using mutually synchronized oscillator arrays," IEEE Traps. Microwave Theory Tech., vol. 39, pp. 1000-1009, June 1991.

[5] J. A. Navarro, Y.-H. Shu, and K. Chang, "Broadband electronically tunable planar active radiating elements and spatial power combiners using notch antennas," IEEE Trans. Microwave Theory Tech., vol. 40, pp. 323-328, Feb. 1992.

[6] J. Birkeland and T. Itoh, "A 16-element quasi-optical FET oscillator power-combing array with external injection locking," IEEE Trans. Microwave Theory Tech., vol. 40, pp. 475-481, Mar. 1992.

[7] S. Battiboia, A. Caliumi, S. Catena, E. Marazzi, and L. Masini, "Lowpower $X$-band radar for indoor burglar alarms," IEEE Trans. Microwave Theory Tech., vol. 43, pp. 1710-1714, July 1995.

[8] D. M. Pozar, "Considerations for millimeter wave printed antennas," IEEE Trans. Antennas Propagat., vol. AP-31, pp. 740-747, Sept. 1983

[9] R. Q. Lee, T. Huynh, and K. F. Lee, "Experimental study of the cross-polarization characteristics of rectangular microstrip antennas," Microwave Opt. Technol. Lett., vol. 2, pp. 247-249, July 1989.

[10] B. K. Kormanyos, W. Harokopus, L. P. B. Katehi, and G. M. Rebeiz, "CPW-fed active slot antennas," IEEE Trans. Microwave Theory Tech. vol. 42, pp. 541-545, Apr. 1994.
[11] C.-K. C. Tzuang and G.-J. Chou, "An active microstrip leaky-wave antenna employing uniplanar oscillator," in Proc. 25 th European Microwave Conf., 1995, pp. 308-311.

[12] A. A. Oliner, "A new class of scannable millimeter wave antennas," in Proc. 20th European Microwave Conf., 1990, pp. 95-104.

[13] A. A. Oliner and K. S. Lee, "Microstrip leaky-wave strip antennas," in IEEE Int. Antennas Propagat. Symp. Dig., pp. 443-446, 1986.

[14] T. Hirota, Y. Tarusawa, and H. Ogawa, "Uniplanar MMTC hybrids-A proposed new MMIC structure," IEEE Trans. Microwave Theory Tech., vol. MTT-35, pp. 576-581, June 1987.

[15] K. C. Gupta, "CAD of active microstrip antennas and microstrip arrays," in Proc. 7th Asia-Pacific Microwave Conf., 1994, pp. 891-895.

[16] K. S. Lee, "Microstrip line leaky-wave antenna,", Ph.D. dissertation, Polytechnic Instit., Brooklyn, New York, 1986.

[17] T. Itoh, "Spectral domain immittance approach for dispersion characteristics of generalized printed transmission lines," IEEE Trans. Microwave Theory Tech., vol. MTT-28, pp. 733-736, July 1980.

[18] C. A. Balanis, Antenna Theory Analysis and Design, New York: Wiley, 1982.

[19] G.-J. Chou and C.-K. C. Tzuang, "An integrated quasi-planar leakywave antenna," IEEE Trans. Antennas Propagat, to be published.

[20] C.-H. Ho, L. Fan, and K. Chang, "Experimental investigations of CPWslotline transitions of uniplanar microwave-integrated circuits," in IEEE MTT-S Int. Microwave Symp. Dig., pp. 877-880, 1993.

[21] H. K. Smith and P. E. Mayes, "Log-periodic array of dual-feed microstrip patch antennas," IEEE Trans. Antennas Propagat., vol. 39, pp. 1659-1664, Dec. 1991.

[22] K. M. Johnson, "Large-signal GaAs MESFET oscillator design," IEEE Trans. Microwave Theory Tech, vol. MTT-27, pp. 217-227, Mar. 1979.

[23] G. L. Matthaei, L. Young, and E. M. T. Jones, Microwave Filters, Impedance-Matching Networks, and Coupling Structures. New York: McGraw-Hill, 1964.

[24] Y. Tajima and K. Mishima, "Transmission-type injection locking of GaAs Schottky-barrier FET oscillators," IEEE Trans. Microwave Theory Tech., vol. MTT-27, pp. 386-391, May 1979.

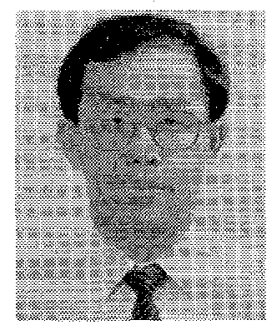

Guang-Jong Chou was born in Pong-Hu, Taiwan, R.O.C., on July 19,1960 . He received the B.S.E.E. degree from Chung Cheng Institute of Technology, Taiwan, and the M.S. degree in communication engineering from National Chiao Tung University, Hsinchu, Taiwan, in 1983 and 1988, respectively. He is currently pursuing the Ph.D. degree at National Chiao Tuang University.

From 1983 to 1993 , he was with the Chung Shang Institute of Science and Technology, LungTan, Taiwan, where he was involved in the design and maintenance of an IFF system. His current research interests include microwave and millimeter wave-integrated circuit design.

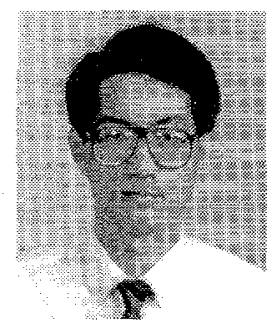

Ching-Kuang $C$. Truang (S'84-M'86-SM'92) was born in Taiwan, R.O.C. on May 10, 1955. He received the B.S. degree in electronic engineering from National Chiao Tung University, Hsinchu, Taiwan, the M.S. degree from the University of California, Los Angeles, and the Ph.D. degree in electrical engineering from the University of Texas at Austin in 1977, 1980, and 1986, respectively.

From February 1981 to June 1984 , he was with TRW, Redondo Beach, CA, working on analog and digital monolithic microwave-integrated circuits. In 1986, he worked on high-speed transient analyses of monolithic microwaveintegrated circuits at UT-Austin. Since September 1986, he has been with the Institute of Communication Engineering, National Chiao Tung University. His research activities involve the design and development of millimeter-wave and microwave-active passive circuits, field theory analysis, and the design of various quasi-optical-integrated antennas. 